\title{
Minimum Resistance Anisotropy of Epitaxial Graphene on SiC
}

\author{
D. Momeni Pakdehi, ${ }^{* \dagger}$ ¡๑ J. Aprojanz, ${ }^{\ddagger}$ A. Sinterhauf, ${ }^{\S, \perp}$ K. Pierz, ${ }^{* \dagger}$ M. Kruskopf, ${ }^{\dagger}$ P. Willke, ${ }^{\S}$ \\ J. Baringhaus, ${ }^{\ddagger}$ J. P. Stöckmann, ${ }^{\ddagger}$ G. A. Traeger, ${ }^{\S}$ F. Hohls, ${ }^{\dagger}$ C. Tegenkamp, $,{ }^{\ddagger}, \|$ M. Wenderoth, ${ }^{\S, \perp}$ \\ F. J. Ahlers, ${ }^{\dagger}$ and H. W. Schumacher ${ }^{\dagger}$ \\ ${ }^{\dagger}$ Physikalisch-Technische Bundesanstalt, Bundesallee 100, 38116 Braunschweig, Germany \\ ${ }^{\ddagger}$ Institut für Festkörperphysik, Leibniz Universität Hannover, Appelstraße 2, 30167 Hannover, Germany \\ ${ }^{\S}$ IV. Physikalisches Institut der Universität Göttingen, Friedrich-Hund-Platz 1, 37077 Göttingen, Germany \\ ${ }^{\perp}$ International Center for Advanced Studies of Energy Conversion (ICASEC) der Universität Göttingen, 37077 Göttingen, Germany \\ "Institute of Physics of Technische Universität Chemnitz, Reichenhainer Straße 70, 09126 Chemnitz, Germany
}

\section{Supporting Information}

ABSTRACT: We report on electronic transport measurements in rotational square probe configuration in combination with scanning tunneling potentiometry of epitaxial graphene monolayers which were fabricated by polymer-assisted sublimation growth on $\mathrm{SiC}$ substrates. The absence of bilayer graphene on the ultralow step edges of below $0.75 \mathrm{~nm}$ scrutinized by atomic force microscopy and scanning tunneling microscopy result in a not yet observed resistance isotropy of graphene on $4 \mathrm{H}$ - and $6 \mathrm{H}-\mathrm{SiC}(0001)$ substrates as low as $2 \%$. We combine microscopic electronic properties with nanoscale transport experiments and thereby disentangle the underlying microscopic scattering mechanism to explain the remaining

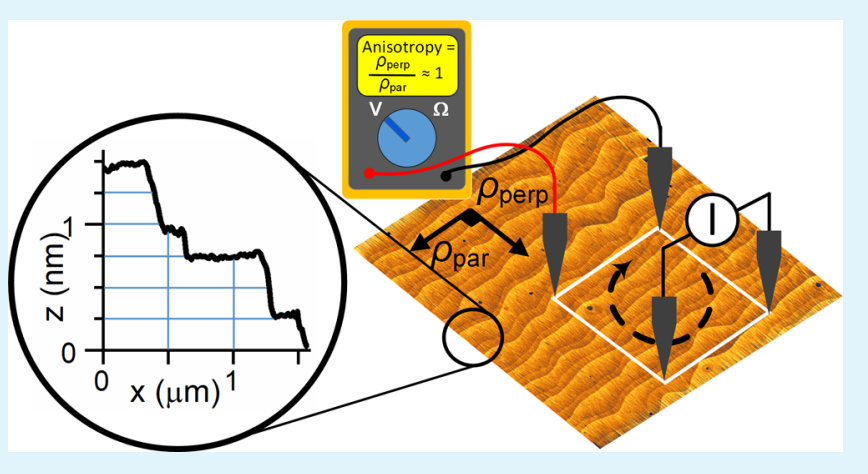
resistance anisotropy. Eventually, this can be entirely attributed to the resistance and the number of substrate steps which induce local scattering. Thereby, our data represent the ultimate limit for resistance isotropy of epitaxial graphene on $\mathrm{SiC}$ for the given miscut of the substrate.

KEYWORDS: SiC epitaxial graphene, isotropic conductance, resistance anisotropy, uniform growth, SiC terrace steps, bilayer-free graphene, scanning tunneling potentiometry

\section{INTRODUCTION}

Epitaxially grown graphene monolayers on $\mathrm{SiC}$ substrates have the potential to be used as a basis for future electronic applications. ${ }^{1-3}$ The main advantage is the capability of waferscale graphene manufacturing directly on the insulating $\mathrm{SiC}$ substrate. Desirable for device fabrication is a high crystal quality over large areas with coherent electronic properties of the graphene layer. However, this is challenging for epitaxial growth. The substrate morphology, in particular $\mathrm{SiC}$ terrace steps, are known to strongly deteriorate the performance of graphene-based electronics, e.g., by limiting the geometry of devices, lowering the cutoff frequency in high-speed electronics, ${ }^{4}$ degrading carrier mobility ${ }^{5}$ in FET devices, ${ }^{6,7}$ or leading to anisotropies in the quantum Hall effect (QHE)., Rotational square probe measurements have quantified a conductance anisotropy of about $70 \%$ for epitaxial graphene layers grown on the Si-face of $6 \mathrm{H}-\mathrm{SiC}{ }^{10}$ Other 4-terminal electronic transport measurements showed a pronounced resistance anisotropy of approximately $60 \%$ and even more than $100 \%$ for epitaxial graphene produced by sublimation growth (SG) methods and chemical vapor deposition (CVD), respectively. $^{7,11}$ In all cases, higher resistance values were observed for transport perpendicular to the $\mathrm{SiC}$ surface terraces, which indicates a correlation with the terrace step edges of the $\mathrm{SiC}$ substrate.

The impact of individual step edges of the substrate on the electrical resistance of the epitaxial graphene layer was investigated by various local scanning tunneling potentiometry (STP) studies which revealed an additional step-induced resistance contribution for charge carrier transport in monolayer graphene across the step edges. ${ }^{12-14}$ Various physical scattering sources were discussed, e.g., detachment from the underlying substrate leading to a potential barrier, induced by a doping variation. ${ }^{14,15}$ Also, local scattering by charge built up, graphene defects, as well as local strain at step edges, were addressed as potential origins. ${ }^{10,16,17}$ Another more considerable contribution arises from the transition region between mono- and bilayer $(\mathrm{ML}-\mathrm{BL})$ graphene due to a wave function mismatch. ${ }^{12,18-20}$ In particular, a ML-BL transition at a $\mathrm{SiC}$ step edge causes a significant increase in the local

Received: December 7, 2017

Accepted: January 29, 2018

Published: January 29, 2018 
Table 1. Samples Used in This Study and the Results from AFM, N4PP Measurements, and STP

\begin{tabular}{|c|c|c|c|c|c|c|c|c|c|c|}
\hline \multirow[b]{2}{*}{ sample } & \multirow[b]{2}{*}{$\begin{array}{c}\text { SiC-polytype/ } \\
\text { miscut }\end{array}$} & \multirow[b]{2}{*}{ process } & \multicolumn{2}{|c|}{ AFM } & \multicolumn{4}{|c|}{ N4PP } & \multicolumn{2}{|c|}{ STP } \\
\hline & & & $h_{\text {step }}(\mathrm{nm})$ & bilayer & $R_{0}(\Omega)$ & $\rho_{\text {par }}(\Omega / \mathrm{sq})$ & $\rho_{\text {perp }}(\Omega / \mathrm{sq})$ & $\begin{array}{l}\text { anisotropy } \\
\text { ratio }\end{array}$ & $\begin{array}{l}\text { anisotropy } \\
\text { ratio }\end{array}$ & $\begin{array}{c}\rho_{\text {sheet }} \\
(\Omega / \text { sq })\end{array}$ \\
\hline S1 & $6 \mathrm{H} / 0.06^{\circ}$ & PASG & $0.25-0.75$ & no & 68 & $629 \pm 1$ & $647 \pm 1$ & $1.03 \pm 0.002$ & $1.03 \pm 0.02$ & $570 \pm 20$ \\
\hline S2 & $4 \mathrm{H} / 0.06^{\circ}$ & PASG & $0.25-1.0$ & no & 67 & $611 \pm 2$ & $620 \pm 2$ & $1.02 \pm 0.005$ & $1.04 \pm 0.02$ & $615 \pm 20$ \\
\hline S3 & $6 \mathrm{H} / 0.06^{\circ}$ & SG & 0.75 & small & 184 & $1755 \pm 13$ & $2046 \pm 15$ & $1.17 \pm 0.01$ & & \\
\hline S4 & $6 \mathrm{H} / 0.37^{\circ}$ & PASG & $0.75-3.5$ & scattered & 112 & $1339 \pm 39$ & $2397 \pm 56$ & $1.79 \pm 0.04$ & & \\
\hline S5 & $6 \mathrm{H} / 0.37^{\circ}$ & $\mathrm{H}_{2} / \mathrm{SG}$ & $3-15$ & extended & 202 & $2121 \pm 48$ & $3531 \pm 54$ & $1.66 \pm 0.03$ & & \\
\hline
\end{tabular}
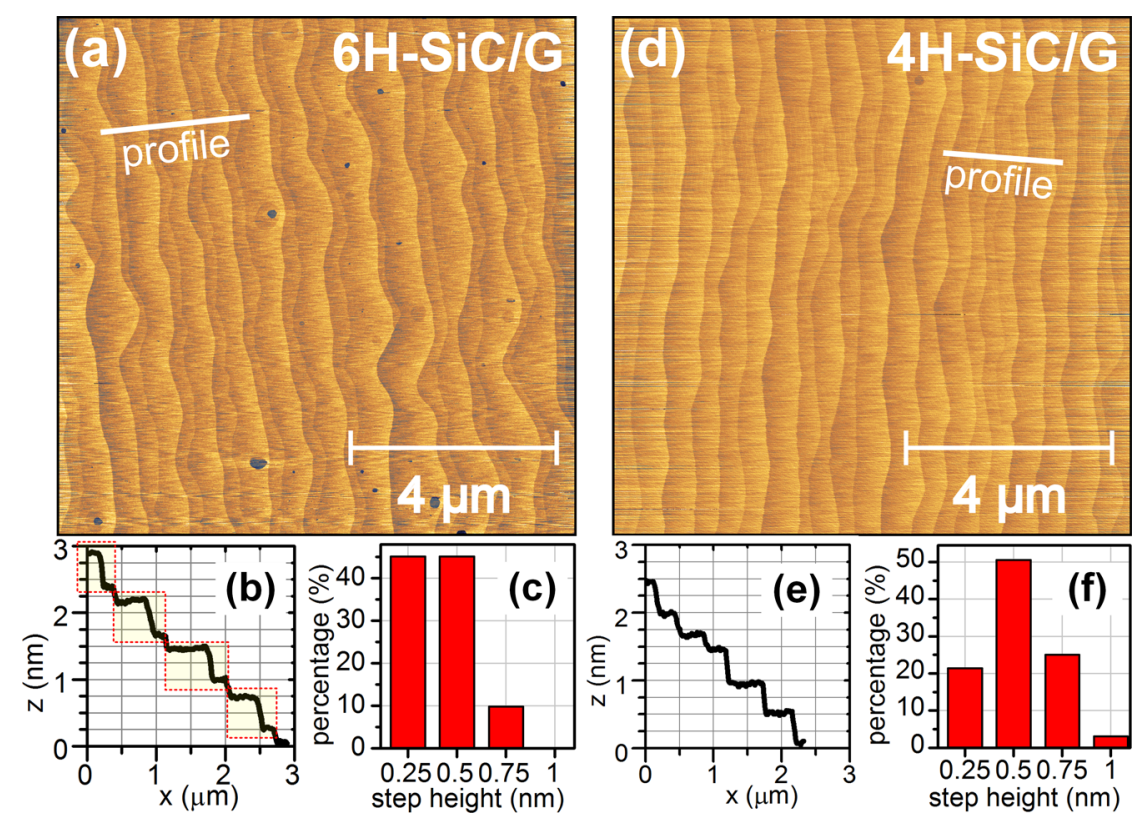

Figure 1. AFM measurements of monolayer graphene grown by the PASG method on $6 \mathrm{H}-\mathrm{SiC}$ (sample S1) and $4 \mathrm{H}-\mathrm{SiC}$ (sample S2). (a) Surface topography of S1. (b) Height profile along the profile line in panel a showing the pairwise sequence of 0.25 and $0.5 \mathrm{~nm}$ steps (marked by red dotted rectangles) typical when using $6 \mathrm{H}-\mathrm{SiC}$ substrates. (c) Statistical evaluation of nine AFM images from the center, edges, and corners of the sample indicating the remarkable homogeneity all over the sample. (d) Surface topography of S2 using 4H-SiC substrates as well as (e) the corresponding height profile and (f) the step height distribution.

resistance. Moreover, magnetotransport measurements in bilayer-patched monolayer graphene showed that bilayers could cause anomalies in the quantum Hall effect. ${ }^{21}$ The influence of bilayer regions on charge magnetotransport also depends on the bilayer position and its carrier density, which later determines the metallic or insulating behavior of the bilayer. Accordingly, magnetotransport in graphene can be interfered, either shunted by the bilayer or constricted through the monolayer graphene regions in case of metallic or insulating bilayer's characteristic, respectively. ${ }^{22}$ This suggests that bilayers can have a substantial impact on the transport properties of graphene devices, and an impact on the resistance anisotropy is expected. Because the formation of bilayer graphene is very often observed at step edges higher than three Si-C bilayers, ${ }^{23,24}$ it is highly favorable to keep $\mathrm{SiC}$ step heights below $0.75 \mathrm{~nm}$ to prevent bilayer formation during epitaxial graphene growth.

In this study, we present the successful realization of such ultrasmooth monolayer graphene sheets on $4 \mathrm{H}$-and $6 \mathrm{H}-\mathrm{SiC}$ polytype substrates by the so-called polymer-assisted sublimation growth (PASG) technique. ${ }^{25-27}$ Rotational square probe measurements of the monolayer graphene reveal nearly vanishing resistance anisotropies of only about $3 \%$. This value is in good agreement with the anisotropy determined from STP measurements at individual terrace steps. It can hence be regarded as the ultimate lower limit of resistance anisotropy only given by step induced resistance contributions. This study shows that nearly perfect resistance isotropy of epitaxial graphene sheets can be achieved by careful control of the growth conditions.

\section{SAMPLE PREPARATION}

The growth of epitaxial graphene was performed on the Si-terminated face of $\mathrm{SiC}$ substrates $\left(5 \times 10 \mathrm{~mm}^{2}\right)$ cut from semi-insulating $6 \mathrm{H}$ - and $4 \mathrm{H}$-polytype wafers (nominally $0.06^{\circ}$ toward [11̄00]), in the following referred to as sample S1 and S2, respectively. A low miscut angle of the wafer is an important prerequisite to obtain smooth graphene layers. $^{24,26}$ The epi-ready surface conditioning allows high-quality epitaxial growth without hydrogen pre-etching. A particular growth procedure was applied, including the PASG technique and special temperature ramps, as described in the following, see also Supporting Information. Polymer adsorbates were deposited on the samples by liquid phase deposition from diluted isopropanol-photoresist (AZ5214E) introduced to an ultrasonic bath that was followed by a short isopropanol rinsing, see refs 25 and 26 for details. The subsequent high-temperature growth process was identically carried out on both polytype substrates in a horizontal inductively heated furnace. $^{28}$ Three initial annealing steps at lower temperatures of 900 ${ }^{\circ} \mathrm{C}$ (vacuum, $\left.30 \mathrm{~min}\right), 1200{ }^{\circ} \mathrm{C}$ ( $\mathrm{Ar}$ atmosphere, $\left.900 \mathrm{mbar}, 10 \mathrm{~min}\right)$, and $1400{ }^{\circ} \mathrm{C}$ (Ar atmosphere, $900 \mathrm{mbar}, 2 \mathrm{~min}$ ) were carried out before the graphene growth at $1750{ }^{\circ} \mathrm{C}$ (Ar atmosphere, 900 mbar, 6 $\mathrm{min})$. After the vacuum annealing step, the samples were first allowed 

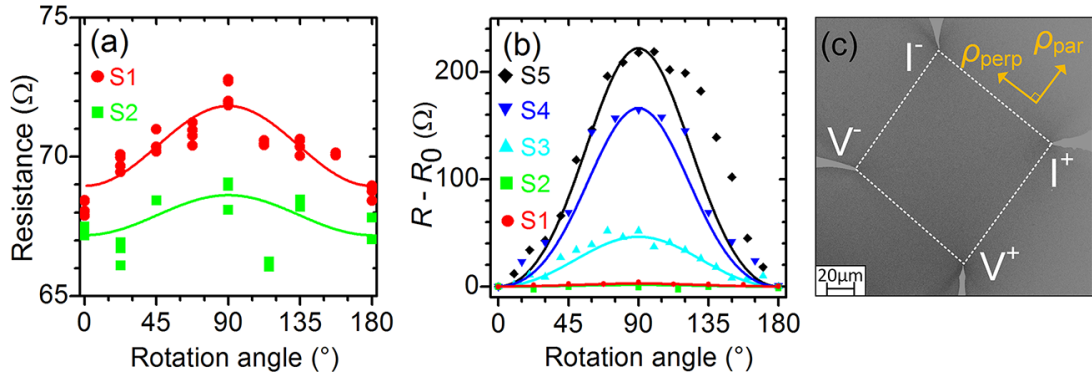

Figure 2. Results from rotational square probe measurements of five epitaxial graphene samples produced under different growth conditions, see Table 1. (a) Resistance variation as a function of the rotation angle for the PASG graphene sample $\mathrm{S} 1$ on $6 \mathrm{H}-\mathrm{SiC}$ and $\mathrm{S} 2$ on $4 \mathrm{H}-\mathrm{SiC}$. (b) Anisotropy related resistance contribution $\left(R-R_{0}\right)$ as a function of the rotation angle of all five graphene samples S1-S5. The fitted curves (solid lines in a and b) are calculated by using a model for anisotropic $2 \mathrm{D}$ sheets, as explained in the literature. ${ }^{32}$ (c) Schematic diagram of the rotational squared N4PP method. The SEM image shows the STM tips on a graphene sample for the N4PP measurement at a rotation angle of $90^{\circ}$.

to cool to room temperature (no argon gas flow ${ }^{25}$ ) for adjusting the pressure to 900 mbar by argon for the later graphene growth. This intermediate cooling for carbon condensation was introduced to increase the number of nucleation sites on the SiC surface for accelerated buffer layer growth.

For comparison, three other graphene samples (S3-S5) were used in this study, listed in Table 1. Graphene sample S3 was grown by conventional sublimation growth (SG) after preannealing in Ar atmosphere $(1000 \mathrm{mbar})$ on a small miscut $6 \mathrm{H}-\mathrm{SiC}$ substrate $\left(\sim 0.06^{\circ}\right) .^{23} \mathrm{~S} 4$ is a PASG graphene sample on a $6 \mathrm{H}-\mathrm{SiC}$ substrate with a large miscut angle of $\sim 0.37^{\circ} .{ }^{26}$ The graphene of S5 was fabricated by sublimation growth on a hydrogen pre-etched $6 \mathrm{H}-\mathrm{SiC}$ substrate. ${ }^{23}$ The main parameters $\left(1750{ }^{\circ} \mathrm{C}, \sim 1\right.$ bar Ar atmosphere, 6 $\mathrm{min}$ ) of the graphene growth were kept the same for all samples.

\section{RESULTS AND DISCUSSION}

Surface Morphology. The atomic force microscopy (AFM) topography images of the graphene monolayers grown on $6 \mathrm{H}$ - and $4 \mathrm{H}-\mathrm{SiC}$ substrates, samples $\mathrm{S} 1$ and $\mathrm{S} 2$, are shown in Figures 1a and d. The very smooth and homogeneous surface morphology is a typical result and can be found on the entire surface of the samples. This is confirmed by multiple AFM measurements at different positions in the center and near the edges of the samples, as well as by optical microscopy inspection throughout the surface. The corresponding histograms in Figures $1 \mathrm{c}$ and $\mathrm{f}$ are the results of AFM inspection of about 200 steps collected from 9 different positions on the substrates, including edge regions. For most of the terrace steps on both polytypes, we found heights below $0.75 \mathrm{~nm}$.

A closer inspection of the topography in Figure 1a reveals a regular and alternating sequence of terraces with a $0.25 \mathrm{~nm}$ high step in front of a terrace with $0.5 \mathrm{~nm}$ step-height for the $6 \mathrm{H}-\mathrm{SiC}$ sample. This situation is depicted in the height profile of Figure $1 b$. The clear majority of the terrace steps ( $90 \%)$ exhibit such a sequential pattern and only occasionally (10\%) steps with $0.75 \mathrm{~nm}$ height are observed, see the histogram in Figure 1c. Higher steps were not found, which confirms that step bunching is effectively suppressed by the PASG technique.

For the graphene on the $4 \mathrm{H}-\mathrm{SiC}$ polytype, no such repeating sequence of steps is observed, Figure 1e. The step height histogram in Figure if shows a different and somewhat wider height distribution compared to the $6 \mathrm{H}$ polytype. Although the majority $(50 \%)$ of steps are $0.5 \mathrm{~nm}$ high as before, a smaller percentage $(20 \%)$ of $0.25 \mathrm{~nm}$ steps and a higher proportion $(25 \%)$ of $0.75 \mathrm{~nm}$ steps are measured. Here, a tiny portion $(\sim 3 \%)$ of $1 \mathrm{~nm}$ high steps is observed. Nevertheless, the high percentage (70\%) of low steps with heights of 0.25 and $0.5 \mathrm{~nm}$ is remarkable and exceeds the results for conventional sublimation growth on $4 \mathrm{H}-\mathrm{SiC}^{24,29}$ Such ultrasmooth graphene layers found on both $\mathrm{SiC}$ polytypes are a unique feature of the PASG technique. It is comparable to graphene layers grown on 3C-SiC(111) surfaces. ${ }^{24} \mathrm{~A}$ second typical property of PASG graphene layers is the suppression of graphene bilayer formation which can be regarded as a result of the very low $\mathrm{SiC}$ step heights $\leq 0.75 \mathrm{~nm}$, in agreement with Raman mappings. ${ }^{25,26}$ The observed formation of the $0.25 / 0.5 \mathrm{~nm}$ step-pairs on the $6 \mathrm{H}-\mathrm{SiC}$ substrate is related to the specific surface-energy sequence of the $\mathrm{SiC}$ bilayer planes of the $6 \mathrm{H}$ polytype. Surface restructuring and step bunching can be understood as retraction of $\mathrm{Si}-\mathrm{C}$ bilayers with different velocities which are related to distinct terrace energies. ${ }^{24,30}$ This retraction process is effectively slowed by the additional carbon supplied from the cracked polymer because the largearea homogeneous carbon nucleation on the terraces accelerates the growth of the buffer layer whose covalent bonds to the $\mathrm{SiC}$ stabilizes the terrace structure. ${ }^{26}$ This enables step bunching only for fast retracting Si-C bilayers, which can catch up a slower one before the surface topography is "frozenin" by the buffer layer. For $6 \mathrm{H}-\mathrm{SiC}$, which has three distinct terrace energies per unit cell, this results in three different retracting velocities and finally to a periodic sequence of 0.25 and $0.5 \mathrm{~nm}$ steps. A similar pattern cannot develop on $4 \mathrm{H}-\mathrm{SiC}$ surfaces which exhibit only two distinct terrace energies per unit cell. ${ }^{24,30}$ However, it is evident that an overall reduction of the step heights is achieved by the PASG technique compared to $\mathrm{SG}$ growth on $4 \mathrm{H}-\mathrm{SiC}$ substrates. ${ }^{24,29}$

Resistance Anisotropy Measurement. The electronic properties of the graphene samples were investigated by angledependent nano four-point probe (N4PP) measurements in an Omicron UHV nanoprobe system. ${ }^{31}$ The samples were kept in $\mathrm{UHV}$ at room temperature after a thermal cleaning procedure by heating up to $300{ }^{\circ} \mathrm{C}$. The scanning tunneling microscopy (STM) tips were placed in a square arrangement with $100 \mu \mathrm{m}$ spacing, and electrical current was flowing between two adjacent tips while the voltage drop was measured between the two opposite ones, Figure 2c. From the ohmic $I-V$-curves which were measured in the current range from -10 to $+10 \mu \mathrm{A}$, the absolute resistance values $R$ were calculated. The N4PP measurements were carried out for different angles between the direction of the current probes and the step edges. The angles of $0^{\circ}$ and $180^{\circ}\left(90^{\circ}\right)$ correspond to current flow parallel (perpendicular) to the steps, and $R_{0}$ denotes the averaged absolute resistance from the parallel $\left(0\right.$ and $\left.180^{\circ}\right)$ measurements, see Table 1 . 

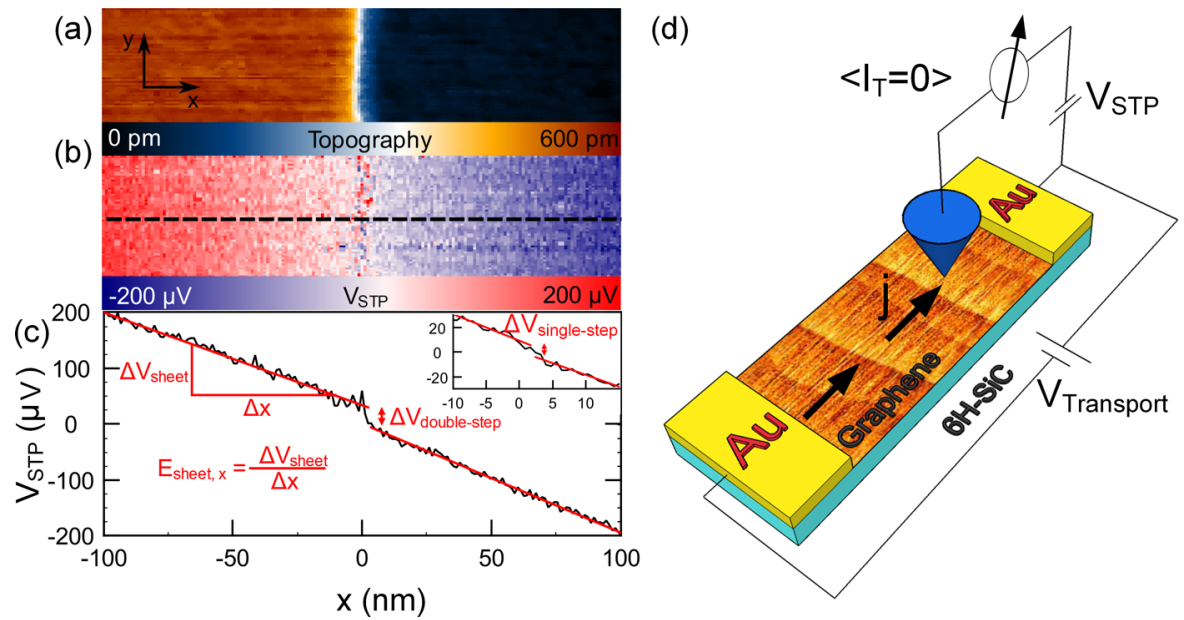

Figure 3. Scanning tunneling potentiometry inspection of step-induced resistance in epitaxial graphene on SiC. (a) Constant current topography of monolayer graphene sheet with a $0.5 \mathrm{~nm}$ step in the center, (tunnel conditions: $I=150 \mathrm{pA}, V_{\text {bias }}=30 \mathrm{mV}$ ). (b) The simultaneously acquired potential map with an average current density of $j=3.6 \mathrm{~A} / \mathrm{m}$. (c) The cross-section along the line in panel b, averaged over all potential values perpendicular to the dashed line in $\mathrm{b}$. The local electric field component in $x$-direction $E_{\text {sheet, } x}$ is calculated from linear fits to the monolayer area (solid red lines in panel c). The step causes an additional local voltage drop $\Delta V \approx 36 \mu \mathrm{V}$. The inset represents the equivalent situation of a monolayer graphene sheet covering a single SiC-bilayer substrate step with a height of $0.25 \mathrm{~nm}$. (d) Schematic of the setup of the scanning tunneling potentiometry experiment, see ref 20 for more details.

The measured resistances $R_{\theta}$ for a given angle $\theta$ are adequately described by

$$
R_{\theta}=\frac{1}{2 \pi \sqrt{\sigma_{\|} \sigma_{\perp}}} \times \ln \sqrt{\frac{\left(\sigma_{\|} / \sigma_{\perp}+1\right)^{2}-4 \cos ^{2} \theta \sin ^{2} \theta\left(\sigma_{\|} / \sigma_{\perp}-1\right)^{2}}{\left(\sin ^{2} \theta+\sigma_{\|} / \sigma_{\perp} \cos ^{2} \theta\right)^{2}}}
$$

, where $\sigma_{\|}$and $\sigma_{\perp}$ denote the conductivities measured parallel and perpendicular to the step direction, respectively, assuming an anisotropic $2 \mathrm{D}$ sheet with different conductivities in $x$ - and $y$-direction. ${ }^{32}$ From the fitting procedure, finally the resistivity values perpendicular $\left(\rho_{\text {perp }}=\sigma_{\perp}^{-1}\right)$ and parallel $\left(\rho_{\text {par }}=\sigma_{\|}^{-1}\right)$ to the step edges are obtained, ${ }^{33}$ and the anisotropy ratio is calculated as $A=\rho_{\text {perp }} / \rho_{\text {par }}$, see Table 1 .

Because the current flow via the semi-insulating $\mathrm{SiC}$ substrate and the buffer layer is negligible, the measured resistance is related to the $2 \mathrm{D}$ graphene sheet on top. For the applied rotational square method, it was shown that it is sensitive to both, a possible intrinsic anisotropy of the graphene, and additional superimposed effects (extrinsic anisotropy), e.g., step edges. $^{32}$ Due to the isotropic dispersion of the density of states near the Fermi level, an isotropic resistivity for graphene is expected. ${ }^{10,34}$ Any measured anisotropy is therefore related to extrinsic effects.

Figure 2a shows the measured resistance $R$ as a function of the rotation angle for the PASG graphene samples S1 and S2. A very slight resistance increase of a few $\mathrm{Ohm}$ is observed at angles around $90^{\circ}$ which indicates that step related effects are noticeable also from these very flat surfaces. However, they are of minimal impact on the resistance anisotropy which is expressed by the obtained very small values of $A_{\mathrm{S} 1}=1.03$ and $A_{\mathrm{S} 2}=1.02$. This is underlined by the comparison to anisotropies of about 1.7 for epitaxial graphene growth in vacuum using $\mathrm{H}$-etched $\mathrm{SiC}$ substrates. ${ }^{10}$ To understand better the impact of the substrate preparation, N4PP measurements were performed on the other samples S3 and S4.

Figure $2 \mathrm{~b}$ shows the anisotropy related resistance contribution $\left(R-R_{0}\right)$ as a function of the rotation angle of all samples S1-S5. The calculated curves and the experimental data agree very well except for S5, where higher resistance values for angles $>110^{\circ}$ are probably due to tip-induced defects.

The $R-R_{0}$ curves in Figure $2 \mathrm{~b}$ show for samples S3, S4, and S5 an apparent maximum at an angle of $90^{\circ}$, which corresponds to transport perpendicular to the step edges. This indicates that step related sources are responsible for the extrinsic anisotropy in these epitaxial graphene layers. The resistance anisotropy increases to $A_{\mathrm{S} 3}=1.17, A_{\mathrm{S} 4}=1.79$, and $A_{\mathrm{S} 5}=1.66$, respectively. Thus, the values $A_{\mathrm{S} 1}$ and $A_{\mathrm{S} 2}$ of the PASG samples S1 and S2 can be regarded as practically isotropic, which verifies the assumption of an intrinsic isotropy of the graphene monolayer. This also demonstrates that extrinsic effects can be reduced to a level where they practically play no role when refined graphene growth procedures are applied as the presented PASG method on low miscut $4 \mathrm{H}$ - and $6 \mathrm{H}-\mathrm{SiC}$ substrates. The N4PP measurements also show that the resistivity on the terraces is significantly reduced by the PASG method, which is demonstrated by the lower values of $R_{0}$ and $\rho_{\text {par }}$ for $S 1, S 2$, and $S 4$ compared to the other samples. Hall measurements show that this is due to an increased electron mobility, see Supporting Information. ${ }^{25,26}$

Local Resistance Measurements. The assignment of the very small resistance anisotropies of the PASG samples S1 and S2 to step related effects was further investigated by STM and STP measurements at room temperature, which give an insight into the local sheet resistance and the defect resistance induced by substrate steps. ${ }^{12,14,35}$

Figure 3a shows an example of a monolayer graphene sheet crossing a substrate step with a height of $0.5 \mathrm{~nm}$ which is located in the center $(x=0 \mathrm{~nm})$ of the STM topography image taken in an area of $200 \times 50 \mathrm{~nm}$. The accompanied potential jump is clearly visible at the same position in the simultaneously acquired potential map plotted in Figure $3 \mathrm{~b}$. Figure $3 \mathrm{c}$ shows the averaged potential across the flat graphene monolayer regions and the substrate step, from which we deduced the local electric field $E_{\text {sheet }, x}$ in the $x$-direction as well as the voltage drop $\Delta V$ caused by the step. Using the macroscopic current density, we find an almost linear increase in resistances (details on the nomenclature can be found 
elsewhere ${ }^{35}$ ) with step heights: $Q_{1}=4 \pm 2 \Omega \mu \mathrm{m}, \varrho_{2}=10 \pm 2$ $\Omega \mu \mathrm{m}$ and $\varrho_{3}=13 \pm 2 \Omega \mu \mathrm{m}$ for monolayer graphene crossing a substrate step with heights of $0.25,0.5$, or $0.75 \mathrm{~nm}$, respectively, which is in good agreement with literature values. ${ }^{12,14,35}$ The step resistance values are independent of the overall crystal morphology of the $4 \mathrm{H}$ - and $6 \mathrm{H}-\mathrm{SiC}$ surface. The STP results can be compared with the N4PP measurements by setting the additional voltage drop at steps and their relative frequency $c_{\mathrm{i}}$ $[\# / \mu \mathrm{m}]$ in relation to the electric field $\left\langle E_{\text {sheet }}\right\rangle$ on the terraces, accordingly, $A_{\mathrm{STP}}=\left(\left\langle E_{\text {sheet }}\right\rangle+\sum c_{i}\left\langle\Delta V_{i}\right\rangle\right) /\left\langle E_{\text {sheet }}\right\rangle$, resulting in an anisotropy of $1.03 \pm 0.02$ for S1 and $1.04 \pm 0.02$ for S2. The good agreement with the anisotropy value close to 1.0 from the N4PP measurements demonstrates that we reached the ultimate lower limit where the resistance contribution of the substrate steps is the sole cause for the measured anisotropy.

Two implications follow from the linear relation between the step height and the local defect resistance at the step. When using $\mathrm{SiC}$ substrates with a same miscut angle, a similar step related resistance anisotropy value is expected because step density and step height can commensurate each other during the surface restructuring processes. A more significant anisotropy is expected for larger substrate miscut angles which increase the number of steps, its height, or both. These conclusions are valid if only step related resistances in monolayer graphene are considered. Additional extrinsic effects can cause higher resistances and larger anisotropies.

An important source for the resistance anisotropies of our samples S3-S5 is attributed to graphene bilayer domains. Local STP measurements have found that the electronic transition from monolayer to bilayer graphene results in an elevated resistance value which approximately corresponds to that of monolayer graphene over a $0.75 \mathrm{~nm}$ high $\mathrm{SiC}$ step. ${ }^{12-14,20}$ Moreover, when the $\mathrm{ML}-\mathrm{BL}$ transition is accompanied by a topographic height change, the resistance again drastically increases, e.g., by a factor of 4 at a $1 \mathrm{~nm}$ substrate step. ${ }^{12}$ These bilayer related local resistance increase can result in a macroscopic resistance directional dependency, according to the shape and distribution of the bilayer inclusions. Because the bilayer inclusions are not symmetric but show an elongated shape at terraces and are very often embodied as bilayer stripes along the terrace step edges, their presence results in a higher resistance for current flow perpendicular to the terrace step edges compared to current flow on the terraces parallel to the step edges.

For the graphene sample $\mathrm{S} 3$, a larger anisotropy $\left(A_{\mathrm{S} 3}=1.17\right)$ was obtained compared to S1 and S2 $\left(A_{\mathrm{S} 1, \mathrm{~S} 2} \sim 1\right)$ although all were grown on low miscut substrates. As discussed above, this discrepancy is not clear if only step related contributions are considered. The additional resistance anisotropy is attributed to the scattered, micrometer-sized, asymmetric bilayer spots, which are located mainly at the terraces edges of sample S3, see Figures $1 \mathrm{a}$ and $\mathrm{b}$ in ref 23 . This comparison clearly shows that the nearly vanishing resistance anisotropy of the PASG samples $S 1$ and $S 2$ is related to the absence of bilayer graphene.

The significantly increased resistance anisotropy of the samples S4 and S5 compared to S1, S2, and S3 is expected because of the six-times larger $\mathrm{SiC}$ miscut angle. Under the assumption of a linear correlation between step height and step resistance, according to the above-mentioned STP anisotropy equation, one can estimate for pure monolayer graphene an anisotropy of $A_{\mathrm{STP}} \approx 1.2$. The measured anisotropy values of $A_{\mathrm{S} 4}=1.79$ and $A_{\mathrm{S} 5}=1.66$ are much higher and are attributed again to bilayer graphene on the terraces. Both samples show larger bilayer coverages compared to S3, and by taking into account the much higher step concentration in S4 and the giant step edges in S5, respectively, this should drastically increase $\rho_{\text {perp }}$ and the anisotropy. On the other hand, the transport along the terraces can vary, e.g., caused by local planar $\mathrm{ML}-\mathrm{BL}$ transitions. This is reflected by the higher $\rho_{\text {par }}$ value of S5 compared to that of $\mathrm{S} 4$, which results in a smaller anisotropy value, $A_{\mathrm{S} 5}<A_{\mathrm{S} 4}$, although $\rho_{\text {perp }}$ of S5 shows the highest value of all samples. This is probably due to the very high terrace steps in S5, which cause extensive graphene bilayer stripes along the upper side of the step edges.

\section{CONCLUSION}

In summary, we studied the resistance anisotropy in epitaxial graphene grown by different sample preparation and growth methods on $4 \mathrm{H}$ - and $6 \mathrm{H}-\mathrm{SiC}(0001)$ substrates with small and large miscut angles. In agreement with STP measurements, the rotational square probe measurements reveal very small resistance anisotropies of $\sim 3 \%$ for graphene layers grown by PASG on $\mathrm{SiC}$ substrates with a small miscut angle. This anisotropy value is traced back to the step resistances of the monolayer graphene across the $\mathrm{SiC}$ steps measured by STP on the nanoscale. The main reason for the vanishing small resistance anisotropy was identified to be the absence of bilayer domains while the specific step resistances are similar to other graphene. The PASG and fine growth optimization methods allow the uniform fabrication of ultrasmooth graphene with most of the terrace step edges being $0.5 \mathrm{~nm}$ or lower, which prevent the formation of graphene bilayer domains. In particular, on the $6 \mathrm{H}-\mathrm{SiC}$ substrate, a very high percentage of $90 \%$ is achieved with a typical pattern of alternating steps of 0.25 and $0.5 \mathrm{~nm}$ in height, which is related to the $\mathrm{SiC}$ layer sequence in this polytype. This study shows that graphene growth using the PASG method and fine-tuning of the growth parameters bears the potential to reduce the terrace step heights down to an ultimate level of a single Si-C bilayer. Because $\mathrm{SiC}$ substrate steps cannot be entirely avoided, it is impossible to achieve perfect resistance isotropy for epitaxial graphene. However, for the produced bilayer-free graphene on ultralow terraces, negligible small deviations from isotropy can be obtained. In general, this study highlights the importance of bilayer-free graphene growth for all kinds of epitaxial growth techniques, whenever isotropic properties are demanded for perfect device performance. It makes the device orientation independent of step direction and improves the freedom for designing device layouts, thereby promoting the potential for future device applications of epitaxial graphene.

\section{ASSOCIATED CONTENT}

\section{S Supporting Information}

The Supporting Information is available free of charge on the ACS Publications website at DOI: 10.1021/acsami.7b18641.

Graphene growth, electronic transport, QHE measurement, and Raman spectroscopy on ultrasmooth graphene monolayer (PDF)

\section{AUTHOR INFORMATION}

\section{Corresponding Authors}

*E-mail: davood.momeni.pakdehi@ptb.de.

*E-mail: klaus.pierz@ptb.de.

ORCID

D. Momeni Pakdehi: 0000-0001-8051-2011 


\section{Notes}

The authors declare no competing financial interest.

\section{ACKNOWLEDGMENTS}

D.M.P. acknowledges support from the School for Contacts in Nanosystems (NTH nano). M.K. acknowledges support from the Braunschweig International Graduate School of Metrology (B-IGSM) and NanoMet. J.A. and J.P.S. thank the support from DFG project Te386/12-1, and P.W. acknowledges the support from DFG priority program 1459 Graphene.

\section{REFERENCES}

(1) Berger, C.; Song, Z.; Li, T.; Li, X.; Ogbazghi, A. Y.; Feng, R.; Dai, Z.; Marchenkov, A. N.; Conrad, M. E. H.; First, P. N.; De Heer, W. A. Ultrathin Epitaxial Graphite: 2D Electron Gas Properties and a Route toward Graphene-Based Nanoelectronics. J. Phys. Chem. B 2004, 108 (52), 19912-19916.

(2) First, P. N.; de Heer, W. A.; Seyller, T.; Berger, C.; Stroscio, J. A.; Moon, J.-S. Epitaxial Graphenes on Silicon Carbide. MRS Bull. 2010, 35 (4), 296-305.

(3) Avouris, P.; Dimitrakopoulos, C. Graphene: Synthesis and Applications. Mater. Today 2012, 15 (3), 86-97.

(4) Lin, Y.-M. M.; Farmer, D. B. B.; Jenkins, K. A. A.; Wu, Y.; Tedesco, J. L. L.; Myers-Ward, R. L. L.; Myers-Ward, C. R.; Gaskill, D. K. K.; Dimitrakopoulos, C.; Avouris, P. Enhanced Performance in Epitaxial Graphene FETs with Optimized Channel Morphology. IEEE Electron Device Lett. 2011, 32 (10), 1343-1345.

(5) Tan, Y. W.; Zhang, Y.; Bolotin, K.; Zhao, Y.; Adam, S.; Hwang, E. H.; Das Sarma, S.; Stormer, H. L.; Kim, P. Measurement of Scattering Rate and Minimum Conductivity in Graphene. Phys. Rev. Lett. 2007, 99 (24), 10-13.

(6) Odaka, S.; Miyazaki, H.; Li, S. L.; Kanda, A.; Morita, K.; Tanaka, S.; Miyata, Y.; Kataura, H.; Tsukagoshi, K.; Aoyagi, Y. Anisotropic Transport in Graphene on SiC Substrate with Periodic Nanofacets. Appl. Phys. Lett. 2010, 96 (6), 62111.

(7) Kobayashi, K.; Tanabe, S.; Tao, T.; Okumura, T.; Nakashima, T.; Aritsuki, T.; O, R. S.; Nagase, M. Resistivity Anisotropy Measured Using Four Probes in Epitaxial Graphene on Silicon Carbide. Appl. Phys. Express 2015, 8 (3), 036602.

(8) Schumann, T.; Friedland, K.-J.; Oliveira, M. H.; Tahraoui, a.; Lopes, J. M. J.; Riechert, H. Anisotropic Quantum Hall Effect in Epitaxial Graphene on Stepped SiC Surfaces. Phys. Rev. B: Condens. Matter Mater. Phys. 2012, 85 (23), 235402.

(9) Pallecchi, E.; Lafont, F.; Cavaliere, V.; Schopfer, F.; Mailly, D.; Poirier, W.; Ouerghi, a. High Electron Mobility in Epitaxial Graphene on $4 \mathrm{H}-\mathrm{SiC}(0001)$ via Post-Growth Annealing under Hydrogen. Sci. Rep. 2015, 4 (1), 4558.

(10) Yakes, M. K.; Gunlycke, D.; Tedesco, J. L.; Campbell, P. M.; Myers-Ward, R. L.; Eddy, C. R; Gaskill, D. K.; Sheehan, P. E.; Laracuente, A. R. Conductance Anisotropy in Epitaxial Graphene Sheets Generated by Substrate Interactions. Nano Lett. 2010, 10 (5), $1559-1562$.

(11) Ciuk, T.; Cakmakyapan, S.; Ozbay, E.; Caban, P.; Grodecki, K.; Krajewska, A.; Pasternak, I.; Szmidt, J.; Strupinski, W. Step-EdgeInduced Resistance Anisotropy in Quasi-Free-Standing Bilayer Chemical Vapor Deposition Graphene on SiC. J. Appl. Phys. 2014, 116 (12), 123708.

(12) Ji, S.; Hannon, J. B.; Tromp, R. M.; Perebeinos, V.; Tersoff, J.; Ross, F. M. Atomic-Scale Transport in Epitaxial Graphene. Nat. Mater. 2011, 11 (2), 114-119.

(13) Clark, K. W.; Zhang, X. G.; Vlassiouk, I. V.; He, G.; Feenstra, R. M.; Li, A. P. Spatially Resolved Mapping of Electrical Conductivity across Individual Domain (Grain) Boundaries in Graphene. ACS Nano 2013, 7 (9), 7956-7966.

(14) Willke, P.; Kotzott, T.; Pruschke, T.; Wenderoth, M. Magnetotransport on the Nano Scale. Nat. Commun. 2017, 8, 15283.
(15) Low, T.; Perebeinos, V.; Tersoff, J.; Avouris, P. Deformation and Scattering in Graphene over Substrate Steps. Phys. Rev. Lett. 2012, 108 (9), $1-4$.

(16) Robinson, J.; Weng, X.; Trumbull, K.; Cavalero, R.; Wetherington, M.; Frantz, E.; LaBella, M.; Hughes, Z.; Fanton, M.; Snyder, D. Nucleation of Epitaxial Graphene on SiC (0001). ACS Nano 2010, 4 (1), 153-158.

(17) Nagase, M.; Hibino, H.; Kageshima, H.; Yamaguchi, H. Local Conductance Measurements of Double-Layer Graphene on $\mathrm{SiC}$ Substrate. Nanotechnology 2009, 20 (44), 445704.

(18) Giannazzo, F.; Deretzis, I.; La Magna, a.; Roccaforte, F.; Yakimova, R. Electronic Transport at Monolayer-Bilayer Junctions in Epitaxial Graphene on SiC. Phys. Rev. B: Condens. Matter Mater. Phys. 2012, 86 (23), 235422.

(19) Clark, K. W.; Zhang, X. G.; Gu, G.; Park, J.; He, G.; Feenstra, R. M.; Li, A. P. Energy Gap Induced by Friedel Oscillations Manifested as Transport Asymmetry at Monolayer-Bilayer Graphene Boundaries. Phys. Rev. X 2014, 4 (1), 1-12.

(20) Willke, P.; Druga, T.; Ulbrich, R. G.; Schneider, M. A.; Wenderoth, M. Spatial Extent of a Landauer Residual-Resistivity Dipole in Graphene Quantified by Scanning Tunnelling Potentiometry. Nat. Commun. 2015, 6, 6399.

(21) Iagallo, A.; Tanabe, S.; Roddaro, S.; Takamura, M.; Sekine, Y.; Hibino, H.; Miseikis, V.; Coletti, C.; Piazza, V.; Beltram, F.; Heun, S. Bilayer-Induced Asymmetric Quantum Hall Effect in Epitaxial Graphene. Semicond. Sci. Technol. 2015, 30 (5), 1-6.

(22) Chua, C.; Connolly, M.; Lartsev, A.; Yager, T.; Lara-Avila, S.; Kubatkin, S.; Kopylov, S. V.; Fal'Ko, V.; Yakimova, R.; Pearce, R.; Janssen, T. J. B. M.; Tzalenchuk, A.; Smith, C. G. Quantum Hall Effect and Quantum Point Contact in Bilayer-Patched Epitaxial Graphene. Nano Lett. 2014, 14 (6), 3369-3373.

(23) Kruskopf, M.; Pierz, K.; Wundrack, S.; Stosch, R.; Dziomba, T.; Kalmbach, C. C.; Müller, A.; Baringhaus, J.; Tegenkamp, C.; Ahlers, F. J.; Schumacher, H. W. Epitaxial Graphene on SiC: Modification of Structural and Electron Transport Properties by Substrate Pretreatment. J. Phys.: Condens. Matter 2015, 27 (18), 185303.

(24) Yazdi, G. R.; Vasiliauskas, R.; Iakimov, T.; Zakharov, A.; Syväjärvi, M.; Yakimova, R. Growth of Large Area Monolayer Graphene on $3 \mathrm{C}-\mathrm{SiC}$ and a Comparison with Other $\mathrm{SiC}$ Polytypes. Carbon 2013, 57, 477-484.

(25) Momeni Pakdehi, D. Epitaxial Graphene on $4 \mathrm{H}$ - and $6 \mathrm{H}-\mathrm{SiC}$ : Growth Optimization and Characterization. M.Sc. Thesis, Bremen City University of Applied Sciences, Bremen, Germany, 2015.

(26) Kruskopf, M.; Momeni Pakdehi, D.; Pierz, K.; Wundrack, S.; Stosch, R.; Dziomba, T.; Götz, M.; Baringhaus, J.; Aprojanz, J.; Tegenkamp, C.; Lidzba, J.; Seyller, T.; Hohls, F.; Ahlers, F. J.; Schumacher, H. W. Comeback of Epitaxial Graphene for Electronics: Large-Area Growth of Bilayer-Free Graphene on SiC. 2D Mater. 2016, 3 (4), 1-9.

(27) Kruskopf, M.; Pierz, K. Verfahren zum Herstellen von Graphen, European patent EP3106432(A1), 2016.

(28) Ostler, M.; Speck, F.; Gick, M.; Seyller, T. Automated Preparation of High-Quality Epitaxial Graphene on $6 \mathrm{H}-\mathrm{SiC}(0001)$. Phys. Status Solidi B 2010, 247 (11-12), 2924-2926.

(29) Hassan, J.; Virojanadara, C.; Meyer, A.; Ivanov, I. G.; Flege, J. I.; Watcharinyanon, S.; Falta, J.; Johansson, L. I.; Janzén, E. Surface Evolution of $4 \mathrm{H}-\mathrm{SiC}(0001)$ during in-Situ Surface Preparation and Its Influence on Graphene Properties. Mater. Sci. Forum 2012, 717-720 (1), 605-608.

(30) Chien, F. R.; Nutt, S. R.; Yoo, W. S.; Kimoto, T.; Matsunami, H. Terrace Growth and Polytype Development in Epitaxial $\beta$-SiC Films on $\alpha$-SiC (6H and 15R) Substrates. J. Mater. Res. 1994, 9 (4), 940954.

(31) Baringhaus, J.; Edler, F.; Neumann, C.; Stampfer, C.; Forti, S.; Starke, U.; Tegenkamp, C. Local Transport Measurements on Epitaxial Graphene. Appl. Phys. Lett. 2013, 103 (11), 111604.

(32) Kanagawa, T.; Hobara, R.; Matsuda, I.; Tanikawa, T.; Natori, A.; Hasegawa, S. Anisotropy in Conductance of a Quasi-One-Dimensional 
Metallic Surface State Measured by a Square Micro-Four-Point Probe Method. Phys. Rev. Lett. 2003, 91 (3), 36805.

(33) Miccoli, I.; Edler, F.; Pfnür, H.; Tegenkamp, C. The 100th Anniversary of the Four-Point Probe Technique: The Role of Probe Geometries in Isotropic and Anisotropic Systems. J. Phys.: Condens. Matter 2015, 27 (22), 223201.

(34) Wallace, P. R. The Band Theory of Graphite. Phys. Rev. 1947, 71 (9), 622-634.

(35) Willke, P.; Schneider, M. A.; Wenderoth, M. Electronic Transport Properties of 1D-Defects in Graphene and Other 2DSystems. Ann. Phys. 2017, 529 (11), 1700003. 